\title{
Influence of material properties and boundary conditions on the dynamic thermal behaviour of a building corner
}

\author{
J. Prata ${ }^{1,2}$, A. Tadeu ${ }^{1,2} \&$ N. Simões ${ }^{1,2}$ \\ ${ }^{1}$ CICC, Department of Civil Engineering, \\ University of Coimbra, Portugal \\ ${ }^{2}$ ITeCons - Institute for Research and Technological Development in \\ Construction Sciences, Portugal
}

\begin{abstract}
Thermal bridges need to be considered in the building design phase to minimize heat loss and prevent building pathologies generated by moisture condensation. The linear thermal bridge (LTB) is normally taken into account at the building design phase, assuming steady-state conditions. However, thermal bridging analysis should involve the variation of temperature over time so as to consider the influence of the thermal inertia of materials. This paper presents the simulation of the transient heat diffusion through a building corner. A 2D numerical modelling tool is used, based on the boundary element method (BEM) and formulated in the frequency domain. This tool enables the calculation of the temperature distribution and the heat flux across the LTB. Several constructive details are analysed, for two different materials (concrete and wood) and with the insulation layer having different placements and thicknesses. Time solutions are obtained afterwards by means of inverse Fourier transformations, thus enabling the simulation of different external temperature variations. A sensitivity analysis is finally performed to analyse the influence of the constructive solution and the boundary conditions on the dynamic thermal behaviour of the LTB.

Keywords: linear thermal bridges, wall corner, heat conduction, boundary element model, boundary conditions, material properties.
\end{abstract}




\section{Introduction}

A linear thermal bridge (LTB) is characterized by a concentration of the heat flow, associated with additional heat loss in winter conditions [1]. It leads to lower surface temperatures, a process that encourages the development of mould and other pathologies caused by condensation [2]. Thermal bridges must therefore be taken into account at the design stage to prevent pathologies and improve building energy efficiency.

In the European Union (EU) the heat balance through linear thermal bridges in residential buildings is taken into account through simplified procedures, assuming a steady state condition. And even whole building dynamic simulation programs used under the Energy Code for service buildings and technical systems, normally assume one-dimensional heat transfer and do not take into account the 2D dynamic heat flows that occur through the linear thermal bridges of building envelopes. However, it might be very important to predict the thermal behaviour of LTBs dynamically at the design stage, assuming temperature variation over time and so considering the influence of the thermal inertia of materials.

Several works have been published that describe different models to assess the dynamic behaviour of thermal bridges. Tadeu et al. [3] proposed a 2D BEM model, formulated in the frequency domain for simulating geometries containing discontinuities such as LTBs. This model was used to calculate the distribution of temperatures and heat flux over time through a building corner, with and without thermal insulation. It was found that a static analysis may underestimate the linear thermal transmittances and may overestimate the surface temperature of the wall in the vicinity of the thermal bridge. Santos et al. [4] used the finite volume method and the MTDMA (MultiTriDiagonal-Matrix Algorithm) to analyse the dynamic hygrothermal behaviour of a building corner by calculating the spatial distribution of temperature, partial vapour pressure and moist air pressure and determining the heat fluxes over time as well as the risk of mould developing due to surface condensation. The results show that near the corner, where the effect of convection and radiation is lower, the risk of these pathologies occurring is higher. Mao and Johannesson [5] developed a tool based on a frequency response approximation method [6] to determine temperatures and heat fluxes within thermal bridges, assuming a linear system and using the finite elements method (FEM) [7].

Other studies report the importance of predicting the thermal behaviour of thermal bridges dynamically $[8,9]$. However, the effective contribution of LTBs to the overall energy performance of a building should be assessed for dynamic conditions and we need to understand how far this contribution depends on the boundary conditions and on the materials properties.

In this paper, the transient heat diffusion through a building corner is simulated using a 2D numerical model, based on the boundary element method (BEM) formulated in frequency domain, following the methodology proposed by Tadeu et al. [3]. Three constructive details are analysed for two different materials (concrete and wood), one without insulation material and the other two with the insulation layer having different placements and thicknesses. Time solutions are obtained afterwards by means of inverse Fourier transformations, thus enabling 
the simulation of different external temperature variations. Climate data from four European cities are used in the simulations. The heat flux across the LTB over time is computed. The results are then compared with the heat flux through a 1D plane wall, computed analytically, to find the influence of the constructive solutions and the boundary conditions on the dynamic thermal behaviour of the LTB.

\section{Numerical methodology}

\subsection{Problem definition}

Consider an LTB at the corner of a building. The surface of the outer-facing wall is subjected to a temperature variation, while the indoor temperature is assumed to be constant. At a certain distance from the corner the heat flux becomes perpendicular to the wall (1D heat flux). So, null heat fluxes can be prescribed along the cut-off sections.

This section describes how the heat transfer by conduction under unsteady state conditions can be computed numerically in a frequency domain. It first presents a BEM formulation to solve the two-dimensional problem. Then, it presents an analytical solution to compute the response at the plane wall, when the problem is $1 \mathrm{D}$ (which occurs at a certain distance from the corner). These two algorithms will later be used together to compute the heat flux at the corner.

\subsection{BEM formulation for an irregular shape}

Consider an unbounded medium (Medium 1) with an embedded inclusion (Medium 2), bounded by a surface $S,\left\{S_{1}, S_{2}, S_{3}\right\} \in S$, as illustrated in Figure 1 .

Null fluxes $(\partial T / \partial n=0)$ are imposed along the boundary section $S_{1}$ and temperatures $T=T_{0}$ are prescribed along section $S_{2}$, while temperatures and heat fluxes are assumed to be stable along the rest of the boundary, $S_{3}$.

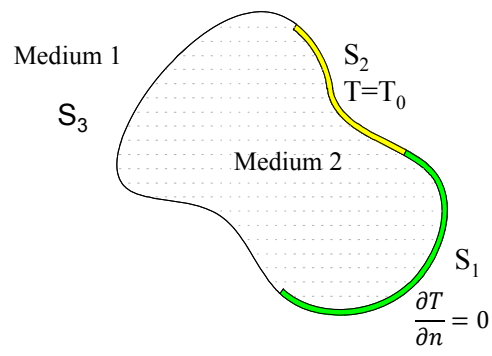

Figure 1: $\quad$ Problem definition. 
The transient heat transfer by conduction is governed by the equation

$$
\left(\frac{\partial^{2}}{\partial x^{2}}+\frac{\partial^{2}}{\partial y^{2}}\right) T=\frac{1}{K} \frac{\partial T}{\partial t}
$$

in which $t$ is time, $T(x, y, t)$ is temperature, $K=k /(\rho c)$ is the thermal diffusivity, $k$ is the thermal conductivity, $\rho$ is the density and $c$ is the specific heat. This formulation assumes that the material's properties are not dependent on the temperature and moisture content.

To solve this equation we move from the time domain to the frequency domain by applying a Fourier transformation in the time domain to eqn. (1) to obtain

$$
\left(\frac{\partial^{2}}{\partial x^{2}}+\frac{\partial^{2}}{\partial y^{2}}+\lambda^{2}\right) \hat{T}(x, y, \omega)=0
$$

where $\hat{T}(x, y, \omega)=\int_{0}^{\infty} T(x, y, t) e^{-\mathrm{i} \omega t} d t, \lambda=\sqrt{\frac{-\mathrm{i} \omega}{K}}, \mathrm{i}=\sqrt{-1}$ and $\omega$ is the frequency. The boundary integral equation can be constructed by applying the reciprocity theorem [10], leading to

a) along the exterior domain of the inclusion (Medium 1):

$$
\begin{aligned}
c \hat{T}^{(1)}\left(x_{0}, y_{0}, \omega\right)= & \int_{S_{3}} q^{(1)}\left(x, y, \eta_{n}, \omega\right) G^{(1)}\left(x, y, x_{0}, y_{0}, \omega\right) d s \\
& -\int_{S_{3}} H^{(1)}\left(x, y, \eta_{n}, x_{0}, y_{0}, \omega\right) \hat{T}^{(1)}(x, y, \omega) d s
\end{aligned}
$$

b) along the interior domain of the inclusion (Medium 2):

$$
\begin{aligned}
& c \hat{T}^{(2)}\left(x_{0}, y_{0}, \omega\right)=\int_{S_{2}+S_{3}} q^{(2)}\left(x, y, \eta_{n}, \omega\right) G^{(2)}\left(x, y, x_{0}, y_{0}, \omega\right) d s \\
& -\int_{S_{1}+S_{3}} H^{(2)}\left(x, y, \eta_{n}, x_{0}, y_{0}, \omega\right) \hat{T}^{(2)}(x, y, \omega) d s \\
& -\int_{S_{2}}^{H_{2}} H^{(2)}\left(x, y, \eta_{n}, x_{0}, y_{0}, \omega\right) \hat{T}_{0}(x, y, \omega) d s
\end{aligned}
$$

In these equations, the superscripts (1) and (2) refer to the exterior and interior domains, $\eta_{\mathrm{n}}$ is the unit outward normal along the boundary, $\mathrm{G}$ and $\mathrm{H}$ are respectively the fundamental solutions (Green's functions) for the temperature $(\widehat{T})$ and heat flux (q), at $(\mathrm{x}, \mathrm{y})$ due to a virtual point heat load at $\left(\mathrm{x}_{0}, \mathrm{y}_{0}\right)$. The factor $c$ is a constant defined by the shape of the boundary, taking the value $1 / 2$ if the shape is smooth and $\left(x_{0}, y_{0}\right) \in S_{1}$ or $\left(x_{0}, y_{0}\right) \in S_{3}$. 
The required Green's functions for temperature and heat flux in Cartesian coordinates are given by

$$
\begin{gathered}
G\left(x, y, x_{0}, y_{0}, \omega\right)=\frac{-\mathrm{i}}{4 k} H_{0}(\lambda r) \\
H\left(x, y, \eta_{n}, x_{0}, y_{0}, \omega\right)=\frac{\mathrm{i}}{4 k} \lambda H_{1}(\lambda r) \frac{\partial r}{\partial \eta_{n}}
\end{gathered}
$$

In which $r=\sqrt{\left(x-x_{0}\right)^{2}+\left(y-y_{0}\right)^{2}}$ and $H_{n}()$ are Hankel functions of the second kind and order $n$.

The final system of equations is assembled so as to ensure the continuity of temperatures and heat fluxes along $S_{3}$. This requires the discretization of the interface $S$ into $N$ straight boundary elements, with one nodal point at the middle of each element $\left(N_{1}, N_{2}\right.$ and $N_{3}$ boundary elements along $S_{1}, S_{2}$ and $S_{3}$, respectively). The final integral equations are manipulated and combined so as to impose the continuity of temperatures and heat fluxes along $S_{3}$, null heat fluxes along $S_{1}$ and prescribed temperatures along $S_{2}$, to establish a system of $\left[\left(N_{1}+N_{2}+2 N_{3}\right) \times\left(N_{1}+N_{2}+2 N_{3}\right)\right]$ equations. The unknown nodal temperatures and heat fluxes are obtained by solving this system of equations so that the heat field in the domain can be defined.

When the element to be integrated is not the loaded element, the integrations in eqns (3) and (4) are evaluated by means of a Gaussian quadrature scheme, using 8 points. For the loaded element, the existing singular integrands in the source terms of the Green's functions are calculated in closed form (see Tadeu et al. [11, 12]), as follows,

$$
\begin{gathered}
\int_{0}^{L / 2} H_{0}(\lambda r) d r=\frac{L}{2} H_{0}\left(\lambda \frac{L}{2}\right)+ \\
+\pi \frac{L}{4}\left[H_{1}\left(\lambda \frac{L}{2}\right) S_{0}\left(\lambda \frac{L}{2}\right)-H_{0}\left(\lambda \frac{L}{2}\right) S_{1}\left(\lambda \frac{L}{2}\right)\right] \\
\int_{0}^{L / 2} H_{1}(\lambda r) \frac{\partial r}{\partial \eta_{n}} d r=0
\end{gathered}
$$

where $S_{n s}(\ldots)$ are Struve functions of order $n S$ and $L$ is the boundary element length. The integrals $\int_{0}^{L / 2} H_{1}(\lambda r) \frac{\partial r}{\partial \eta_{n}} d r$ are zero because $\frac{\partial r}{\partial \eta_{n}}=0$ along the loaded element. 


\subsection{Analytical solutions for layered systems}

Consider a system consisting of a set of $\mathrm{m}$ plane layers of infinite extent, as shown in Figure 3.

The temperatures $t_{0 t}$ and $t_{0 b}$ are imposed for the top and bottom external surfaces. The thermal material properties and thickness of the various layers may differ. The solution is defined in the frequency domain as the superposition of plane heat sources. Thus, the temperatures $T_{0 t}$ and $T_{0 b}$ were first defined by applying a direct discrete fast Fourier transform to the imposed temperature variations in the time domain. As it is assumed that $T_{0 t}$ and $T_{0 b}$ do not vary along $x$, the problem becomes one-dimensional.

The definition of the solution involves establishing surface terms at each interface. The total heat field is found by adding the sets of surface terms arising within each layer and at each interface that are required to satisfy the boundary conditions at the interfaces, i.e. continuity of temperatures and normal fluxes between layers (see [13]).

For the layer $j$, the heat surface terms on the upper and lower interfaces can be expressed as

$$
\begin{aligned}
& \hat{T}_{j 1}(x, y, \omega)=\frac{-\mathrm{i}}{2 k_{j}} \frac{e^{-\mathrm{i} \lambda_{j}\left|y-\sum_{l=1}^{j-1} h_{l}\right|}}{\lambda_{j}} A_{j}^{t} \\
& \hat{T}_{j 2}(x, y, \omega)=\frac{-\mathrm{i}}{2 k_{j}} \frac{e^{-\mathrm{i} \lambda_{j}\left|y-\sum_{l=1}^{j} h_{l}\right|}}{\lambda_{j}} A_{j}^{b}
\end{aligned}
$$

where $\lambda_{j}=\sqrt{-\frac{\mathrm{i} \omega}{K_{j}}}, k_{j}$ is the thermal conductivity, $\rho_{j}$ is the density, $c_{j}$ is the specific heat of the material in the layer $j$ and $h_{l}$ is the thickness of the layer $l$.

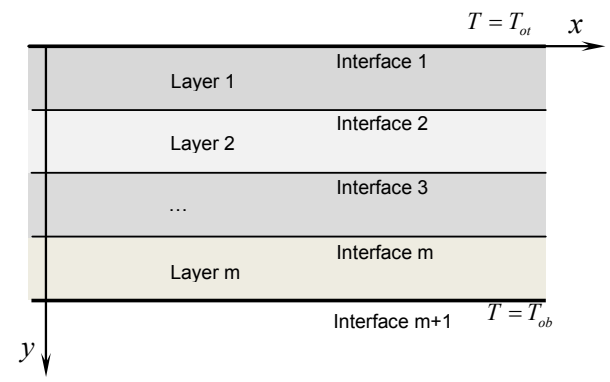

Figure 2: Multilayer system. 
A system of $2 \mathrm{~m}$ equations is derived, ensuring the continuity of temperatures and heat fluxes at each interface:

$$
\left[\begin{array}{ccccc}
\frac{1}{k_{1} \lambda_{1}} & \frac{\mathrm{e}^{-\mathrm{i} \lambda_{1} h_{1}}}{k_{1} \lambda_{1}} & \ldots & 0 & 0 \\
\mathrm{e}^{-\mathrm{i} \lambda_{1} h_{1}} & -1 & \ldots & 0 & 0 \\
\frac{\mathrm{e}^{-\mathrm{i} \lambda_{1} h_{1}}}{k_{1} \lambda_{1}} & \frac{1}{k_{1} \lambda_{1}} & \ldots & 0 & 0 \\
\ldots & \ldots & \ldots & \ldots & \ldots \\
0 & 0 & \ldots & -1 & \mathrm{e}^{-\mathrm{i} \lambda_{m} h_{m}} \\
0 & 0 & \ldots & -\frac{1}{k_{1} \lambda_{m}} & -\frac{\mathrm{e}^{\mathrm{i} \lambda_{m} h_{m}}}{k_{m} \lambda_{m}} \\
0 & 0 & \ldots & \frac{\mathrm{e}^{-\mathrm{i} \lambda_{m} h_{m}}}{k_{m} \lambda_{m}} & \frac{1}{k_{m} \lambda_{m}}
\end{array}\right]\left[\begin{array}{c}
A_{1}^{t} \\
A_{1}^{b} \\
\ldots \\
A_{m}^{t} \\
A_{m}^{b}
\end{array}\right]=\left[\begin{array}{c}
T_{0 t} \\
0 \\
0 \\
\ldots \\
0 \\
0 \\
T_{0 b}
\end{array}\right]
$$

The resolution of this system gives the amplitude of the surface terms at each interface, leading to the following temperature fields at layer $j$ :

$$
\begin{aligned}
\tilde{T}(x, y, \omega)=E_{0 j} & \left(\frac{e^{-\mathrm{i} \lambda_{j}\left|y-\sum_{l=1}^{j-1} h_{l}\right|}}{\lambda_{j}} A_{0 j}^{t}+\frac{e^{-\mathrm{i} \lambda_{j} \mid y-\sum_{l=1}^{j} h_{l}} \mid}{\lambda_{j}} A_{0 j}^{b}\right), \\
& \text { if } \sum_{l=1}^{j-1} h_{l}<y<\sum_{l=1}^{j} h_{l}
\end{aligned}
$$

\subsection{Time domain}

The heat responses in the spatial-temporal domain are obtained by means of an inverse Fourier transform in the frequency domain. In order to prevent the aliasing phenomena, complex frequencies with a small imaginary part of the form $\omega_{c}=\omega-\mathrm{i} \eta$ (with $\eta=0.7 \Delta \omega$, and $\Delta \omega$ being the frequency step) are used in the computation procedure. The constant $\eta$ cannot be made arbitrarily large, since this leads either to a severe loss of numerical accuracy, or to underflows and overflows in the evaluation of the exponential windows.

The time evolution of the temperature amplitude can be varied. The time Fourier transformation of the incident heat field defines the frequency domain where the BEM solution needs to be computed $\hat{T}_{0}(x, y, \omega)=\int_{0}^{\infty} T_{0}(x, y, t) e^{-\mathrm{i} \omega t} d t$. The response needs to be computed from $0.0 \mathrm{~Hz}$ up to very high frequencies. An intrinsic characteristic of this problem is that the heat responses decay very fast as 
the frequency increases, which allows us to limit the upper frequency for the solution.

The final equation is given by

$$
T(x, y, t)=\frac{1}{2 \pi} \int_{0}^{\infty} \hat{T}_{0}(x, y, \omega) \hat{T}(x, y, \omega) e^{\mathrm{i} \omega t} d \omega
$$

which is computed as a discrete inverse fast Fourier transform.

\section{Numerical application}

\subsection{Case studies}

The proposed models were used to simulate heat conduction through an LTB formed at the corner of a building. Three cases are simulated, as illustrated in Figure 3: the wall without thermal insulation (Case 1); the wall with an internal thermal insulation layer (Case 2); the wall with an external thermal insulation layer (Case 3). Two different materials were considered for the wall: concrete (Figure $3 \mathrm{a}$ )) and wood (Figure $3 \mathrm{~b}$ )). The surfaces' thermal resistance is modelled as a thin air layer on the outer surfaces to take into account convection and radiation phenomena. Thermal resistance for the inner surface was considered to be $0.13 \mathrm{~m}^{2} .{ }^{\circ} \mathrm{C} / \mathrm{W}$ and for the outer surface it was $0.04 \mathrm{~m}^{2} .{ }^{\circ} \mathrm{C} / \mathrm{W}$. Three different thicknesses were considered for the thermal insulation layer $(3 \mathrm{~cm}, 6 \mathrm{~cm}$ and $12 \mathrm{~cm}$ ). The corner is modelled as adjacent lateral walls long enough to ensure that the heat transfer at the cut-off planes is not affected by the presence of the thermal
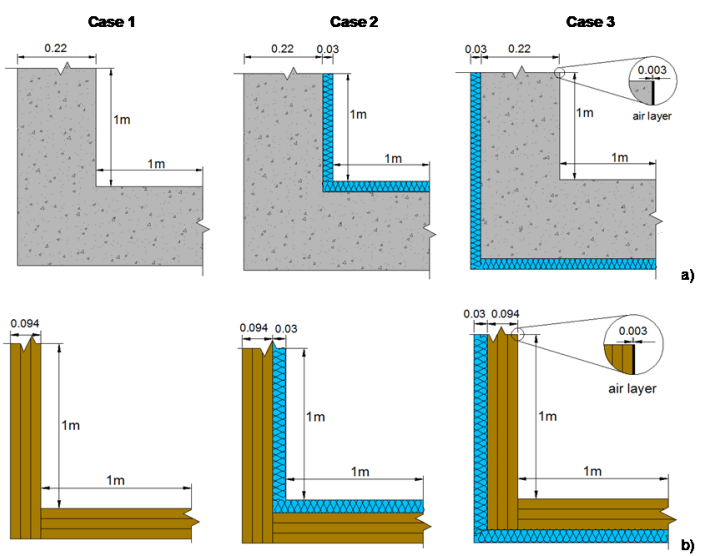

Figure 3: Building corner geometry used in the numerical applications: Case 1 - the wall without external insulation; Case 2 - the wall with an insulating thermal layer on the inside surface; Case 3 - the wall with an insulating thermal layer on the outside surface (dimensions in $\mathrm{m}$ ). a) concrete corner; b) wood corner. 
bridge formed at the junction of the walls. This allows one-dimensional heat fluxes at those sections, which are the cut-off planes. Thus, null fluxes can be prescribed along those cut-off sections, in their normal directions. In the examples, the length of the wall followed ISO Standard 10211:2007 [1], enabling a length of $1 \mathrm{~m}$ to be applied.

The materials' thermal properties are listed in Table 1. The heat loss through the different configurations of the LTB was determined. For this, the global heat flow rate at the inside surface was computed for the whole system, $q_{\text {total }}$. The computations involved integrating the heat fluxes along the inner wall surfaces. $q_{\text {total }}$ includes the heat fluxes associated with the thermal bridge and those crossing the lateral walls $\left(q_{\text {walls }}\right), q_{\text {total }}=q_{\text {LTB }}+q_{\text {walls }} \cdot q_{\text {walls }}$, was then calculated by imposing one-dimensional heat transfer for the lateral walls, using the analytical model proposed. The heat loss through the LTB was obtained by computing the difference between these interior global heat fluxes $q_{\text {LTB }}=q_{\text {total }}-q_{\text {walls }}$.

The influence of the boundary conditions on the dynamic thermal behaviour of the LTB was analysed by performing simulations for different climate data (see section 3.2).

Table 1: Materials' thermal properties.

\begin{tabular}{|c|c|c|c|}
\hline Material & $\begin{array}{c}\text { Conductivity } \\
k \\
\left(\mathrm{~W} \cdot \mathrm{m}^{-1} \cdot{ }^{\mathrm{o}} \mathrm{C}^{-1}\right)\end{array}$ & $\begin{array}{c}\text { Mass density } \\
\rho\left(\mathrm{kg} \cdot \mathrm{m}^{-3}\right)\end{array}$ & $\begin{array}{l}\text { Specific heat } \\
c\left(\mathrm{~J} \mathrm{~kg}^{-1} \cdot{ }^{o} \mathrm{C}^{-1}\right)\end{array}$ \\
\hline Natural cork (NC) & 0.046 & 130.0 & 1638.0 \\
\hline Wood & 0.1 & 500.0 & 2048.0 \\
\hline Concrete & 1.4 & 2300.0 & 880.0 \\
\hline $\begin{array}{l}\text { External surface layer } \\
\quad 3 \mathrm{~mm} \text { thick }\end{array}$ & 0.075 & 1.29 & 1000 \\
\hline $\begin{array}{l}\text { Internal surface layer } \\
3 \mathrm{~mm} \text { thick }\end{array}$ & 0.0231 & 1.29 & 1000 \\
\hline
\end{tabular}

\subsection{Boundary conditions}

The dynamic thermal behaviour of the LTB was analysed for the first 6 days of the year, considering climate data from four European cities (Figure 4): Évora (Portugal), Bragança (Portugal), Amsterdam (Netherlands) and Helsinki (Finland). The average annual heating degree days for each of the cities are: 1150 (Évora), 2015 (Bragança), 2940 (Amsterdam) and 4394 (Helsinki).

At $t=0.0 \mathrm{~h}$, the systems have a uniform temperature of $20^{\circ} \mathrm{C}$ throughout the full domain. Between $t=0.0 \mathrm{~h}$ and $t=48 \mathrm{~h}$ the initial values of the boundary conditions function are kept constant in order to attain steady-state conditions up to the first time step of the dynamic simulations $(t=48 \mathrm{~h})$. The frequency domain of computation ranged from $0.0 \mathrm{~Hz}$ to $4096.0 /(192 \times 3600) \mathrm{Hz}$, with a frequency 

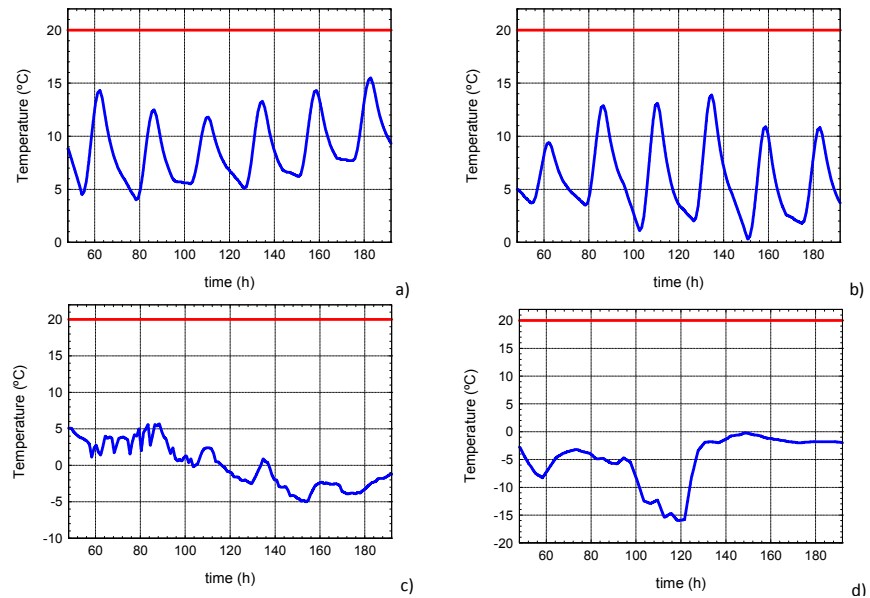

Figure 4: Imposed temperature over time. Climate data related to the first 6 days of the year: a) Évora (Portugal); b) Bragança (Portugal); c) Amsterdam (Netherlands); d) Helsinki (Finland).

increment of $1.0 /(192 \times 3600) \mathrm{Hz}$, which determined a total time window of $192.0 \mathrm{~h}$ for the analysis. When modelled by the BEM the wall's longitudinal material interfaces were discretized using 100 constant boundary elements, while 60 constant boundary elements were used to model the lateral interfaces at the cut-off sections. The temperature distribution is obtained at a very fine two-dimensional grid of receivers equally spaced $0.01 \mathrm{~m}$ apart along the $\mathrm{x}$ and $\mathrm{y}$ directions.

\subsection{Results}

The proposed methodology was used to determine the heat loss through the LTB for each wall configuration (Figure 4). The influence of the constructive solutions and the boundary conditions on the dynamic thermal behaviour of the LTB was analysed by using different climate data (Figure 4) and different thicknesses for the thermal insulation $(3 \mathrm{~cm}, 6 \mathrm{~cm}$ and $12 \mathrm{~cm})$ in the simulations. For each wall configuration, the ratio between total heat loss through the LTB and the total heat loss through $1 \mathrm{~m}^{2}$ of wall surface was computed, taking into account the four climate data sets presented in Figure 4. The results are shown in Figure 5.

It can be seen that the ratio does not significantly depend on the external temperature variation. However, the ratio increases for greater insulation layer thickness and is higher when the insulation layer is applied to the outside of the wall. A mean average ratio was then calculated for each wall configuration (Figure 4), taking into account the results for the different climate data shown in Figure 4 . The average ratio given by the dynamic simulations for each wall configuration were then compared with the results obtained assuming steady-state conditions. A 2D thermal analysis program for steady state heat transfer was used [14]. The results are presented in Figure 6. The average ratio results of the dynamic 

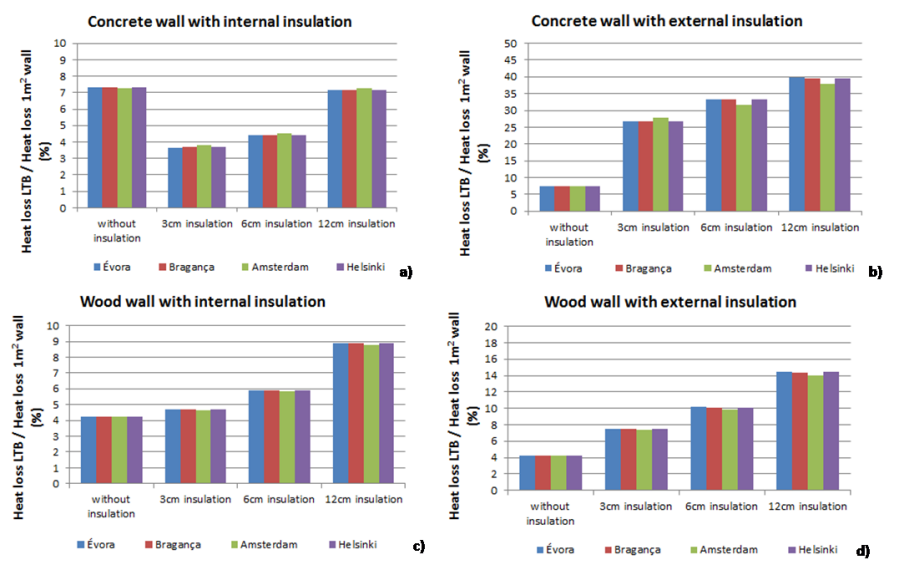

Figure 5: Ratio between the total heat loss though the LTB and the total heat loss though $1 \mathrm{~m}^{2}$ of wall, taking into account the climate data from: Évora, Bragança, Amsterdam and Helsinki.
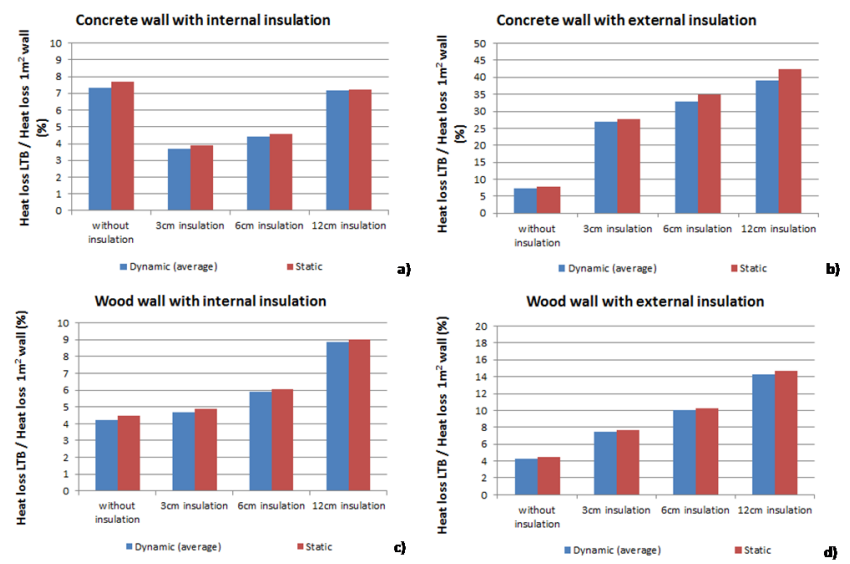

Figure 6: Comparison between the mean average ratio taking into account the results for the different climate data, obtained both under dynamic conditions and assuming steady-state conditions.

numerical simulations for each wall configuration are similar to the ones obtained assuming steady-state conditions. Thus, the ratio between the total heat loss through the LTB and the total heat loss through $1 \mathrm{~m}^{2}$ of wall surface does not depend on the boundary conditions of the problem.

\section{Conclusions}

The simulation of the transient heat diffusion through a building corner has been presented. A 2D numerical modelling tool was used, based on the boundary 
element method (BEM) and formulated in the frequency domain. Several constructive details were analysed for concrete and wood and with the insulation layer having different placements and thicknesses. The heat fluxes through the different constructive details were computed. A sensitivity analysis was performed to analyse de influence of the constructive solutions and the boundary conditions on the dynamic thermal behaviour of the LTB. The results have shown that the ratio between the total heat loss through the LTB and the total heat loss through $1 \mathrm{~m}^{2}$ of wall surface varies with the constructive elements' thermal resistance and with the placement of the insulation layer. However, this ratio does not depend on the boundary conditions of the problem.

\section{Acknowledgements}

The research work presented herein was supported by the Portuguese Foundation for Science and Technology (FCT), under doctoral grant SFRH/BD/91688/2012, by the Initiative Energy for Sustainability (EfS) of the University of Coimbra, and supported by the Energy and Mobility for Sustainable Regions-EMSURE-Project (CENTRO-07-0224-FEDER-002004). This study has also been supported by FEDER funds through COMPETE and QREN, under the project FCOMP-050128-FEDER-022994.

\section{References}

[1] ISO 10211, Thermal bridges in building construction - Heat flows and surface temperatures - Detailed calculations, 2007.

[2] ISO 13788, Hygrothermal performance of building components and building elements -Internal surface temperature to avoid critical surface humidity and interstitial condensation -Calculation methods, 2001.

[3] Tadeu A., Simões I., Simões N. \& Prata J., Simulation of dynamic linear thermal bridges using a BEM model in frequency domain, Energy and Buildings, 43, pp. 3685-3695, 2011.

[4] Santos G.H., Mendes N. \& Philippi P.C., A building corner model for hygrothermal performance and mould growth risk analyses, International Journal of Heat Transfer and Mass Transfer, 52, pp. 4862-4872, 2009.

[5] Mao G., Johannesson G., Dynamic calculation of thermal bridges, Energy and Buildings, 26, pp. 233-240, 1997.

[6] Andersson AC. \& Johannesson G., Application of frequency responses for fast analysis of two-dimensional heat-flow problems, Coden LUTVDDG/(TVBH-7072)/1-8, Lund, Sweden, 1983.

[7] Bathe KJ., Numerical methods in finite element analysis, Englewood Cliffs, NJ: Prentice-Hall, 1976.

[8] Gao Y., Roux JJ., Zhao L.H. \& Jiang Y., Dynamical building simulation: A low order model for thermal bridges losses, Energy and Buildings, 40, pp. 2236-2243, 2008. 
[9] Déqué F., Ollivier F. \& Roux JJ., Effect of 2D modelling of thermal bridges on the energy performance of buildings: numerical application on the Matisse apartment, Energy and Buildings, 33(6), pp. 583-7, 2001.

[10] Wrobel LC. \& Brebbia CA., A formulation of the Boundary Element Method for axisymmetric transient heat conduction, International Journal of Heat and Mass Transfer, 24, pp. 843-850, 1981.

[11] Tadeu A., Santos P. \& Kausel E., Closed-form integration of singular terms for constant, linear and quadratic boundary elements - Part I: SH wave propagation, EABE - Engineering Analysis with Boundary Elements, 23(8), pp. 671-681, 1999.

[12] Tadeu A., Santos P. \& Kausel E., Closed-form integration of singular terms for constant, linear and quadratic boundary elements - Part II: SV-P wave propagation. EABE - Engineering Analysis with Boundary Elements, 23(9), pp. 757-768, 1999.

[13] Tadeu A. \& Simões N., Three-dimensional fundamental solutions for transient heat transfer by conduction in an unbounded Medium, half-space, slab and layered media, Engineering Analysis with Boundary Elements, 30(5), pp. 338-349, 2006.

[14] Physibel-Building Physics Software, BISCO-2D steady state heat transfer free form. Version 9.0w. http://www.physibel.be/ 